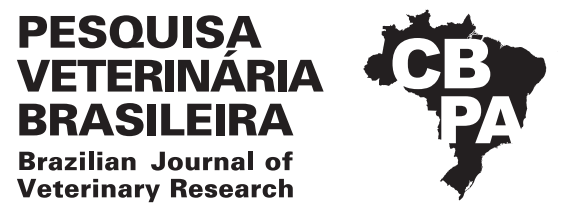

Pesq. Vet. Bras. 38(7):1239-1249, julho 2018 DOI: $10.1590 / 1678-5150-P V B-5548$

Artigo de Revisão

Animais de Produção/Livestock Diseases

ISSN 0100-736X (Print)

ISSN 1678-5150 (Online)

\title{
Plantas cardiotóxicas para ruminantes no Brasil $^{1}$
}

\author{
Naiara C. F. Nascimento ${ }^{2}$, Lorena D.A. Aires ${ }^{3}$, James A. Pfister ${ }^{4}$, \\ Rosane M.T. Medeiros ${ }^{5}$, Franklin Riet-Correa ${ }^{6}$ e Fábio S. Mendonça ${ }^{7 *}$
}

\begin{abstract}
Nascimento N.C.F., Aires L.D.A., Pfister J.A., Medeiros R.M.T., Riet-Correa F. \& Mendonça F.S. 2018. [Cardiotoxic plants affecting ruminants in Brazil.] Plantas cardiotóxicas para ruminantes no Brasil. Pesquisa Veterinária Brasileira 38(7):1239-1249. Laboratório de Diagnóstico Animal, Universidade Federal Rural de Pernambuco, Rua Dom Manoel de Medeiros s/n, Dois Irmãos, Recife, PE 52171-900, Brazil. E-mail: fabio.mendonca@pq.cnpq.br

This review updates information about cardiotoxic plants affecting ruminants in Brazil. Currently it is known that there are at least 131 toxic plants belonging to 79 genera. Twenty five species affect the heart function. Plants that contain sodium monofluoroacetate (Palicourea spp., Psychotria hoffmannseggiana, Amorimia spp., Niedenzuella spp., Tanaecium bilabiatum and Fridericia elegans) cause numerous outbreaks of poisoning, mainly in cattle, but buffaloes, sheep and goats are occasionally affected. Poisoning by Palicourea marcgravii remains the most important due to the wide distribution of this plant in Brazil. New species of the genus Palicourea containing sodium monofluoracetate, such as Palicourea amapaensis, Palicourea longiflora, Palicourea barraensis, Palicourea macarthurorum, Palicourea nigricans, Palicourea vacillans and Palicourea aff. juruana were described in the amazon region. In the northeast region, the most important toxic plant for cattle is Amorimia septentrionalis. In the midwest, outbreaks of Niedenzuella stannea poisoning have been reported in cattle in the Araguaia region and the disease needs to be better investigated for its occurrence and importance. Tetrapterys multiglandulosa and Tetrapterys acutifolia, two plants causing cardiac fibrosis also contain sodium monofluoroacetate and were reclassified to the genus Niedenzuella. These two plants and Ateleia glazioveana, other plant that causes cardiac fibrosis continues to be important in the southeastern and south of Brazil. Other less important are the plants that contain cardiotoxic glycosides, such as Nerium oleander and Kalanchoe blossfeldiana, in wich poisonings are generally accidental. Recently, several experimental methodologies were successfully employed to avoid poisonings by sodium monofluoroacetate containing plants. These methodologies include the induction of food avertion using lithium chloride, the ministration of repeatedly non-toxic doses of leaves to induce resistance, the use of acetamide to prevent poisonings and the intraruminal inoculation of sodium monofluoroacetate degrading bacteria.
\end{abstract}

INDEX TERMS: Poisonous plants, cardiotoxic plants, cardiotoxic glycosides, sodium monofluoroacetate, plant poisoning, ruminants, toxicoses.

\footnotetext{
${ }^{1}$ Recebido em 21 de agosto de 2017.

Aceito para publicação em 28 de agosto de 2017.

2 Programa de Pós-Graduação em Medicina Veterinária, Universidade Federal Rural de Pernambuco (UFRPE), Rua Dom Manoel de Medeiros s/n, Dois Irmãos, Recife, PE 52171-900, Brasil.

${ }^{3}$ Departamento de Medicina Veterinária, Universidade Federal Rural de Pernambuco (UFRPE), Rua Dom Manoel de Medeiros s/n, Dois Irmãos, Recife, PE 52171-900.

${ }^{4}$ Poisonous Plant Research Laboratory, Agricultural Research Service, United States Department of Agriculture, 1150 E. 1400 N., Logan, UT 84341, USA.

${ }^{5}$ Hospital Veterinário, Universidade Federal de Campina Grande (UFCG), Patos, PB 58700-000, Brasil.

${ }^{6}$ Instituto Nacional de Investigacíon Agropecuaria (INIA), La Estanzuela, Colonia, Uruguay, CP 70.000 .

${ }^{7}$ Laboratório de Diagnóstico Animal, Departamento de Morfologia e Fisiologia Animal, Universidade Federal Rural de Pernambuco (UFRPE), Rua Dom Manoel de Medeiros s/n, Dois Irmãos, Recife, PE 52171-900. *Autor para correspondência: fabio.mendonca@pq.cnpq.br
}

RESUMO.- Esta revisão atualiza informações sobre plantas cardiotóxicas que afetam os ruminantes no Brasil. Atualmente, sabe-se que existem pelo menos 131 plantas tóxicas pertencentes a 79 gêneros. Vinte e cinco espécies afetam o funcionamento do coração. As plantas que contêm monofluoroacetato de sódio (Palicourea spp., Psychotria hoffmannseggiana, Amorimia spp., Niedenzuella spp., Tanaecium bilabiatum e Fridericia elegans) causam numerosos surtos de intoxicação, principalmente em bovinos, mas búfalos, ovinos e caprinos são ocasionalmente afetados. A intoxicação por Palicourea marcgravii continua a ser a mais importante devido à ampla distribuição desta planta no Brasil. Novas espécies do gênero Palicourea contendo monofluoracetato de sódio, como Palicourea amapaensis, Palicourea longiflora, Palicourea barraensis, Palicourea 
macarthurorum, Palicourea nigricans, Palicourea vacillans e Palicourea aff. juruana foram descritas na região amazônica. Na região nordeste, a planta tóxica mais importante para bovinos é Amorimia septentrionalis. No Centro-Oeste, surtos de intoxicação por Niedenzuella stannea foram relatados em bovinos na região do Araguaia e a doença precisa ser melhor investigada quanto à sua ocorrência e importância. Tetrapterys multiglandulosa e Tetrapterys acutifolia, duas plantas que causam fibrose cardíaca, também contêm monofluoracetato de sódio e foram reclassificadas para o gênero Niedenzuella. Essas duas espécies e Ateleia glazioveana, outra planta que causa fibrose cardíaca, continuam sendo importantes no Sul e Sudeste do Brasil. Outras espécies menos importantes e que ocasionamente provocam surtos acidentais de intoxicação são as plantas que contém glicosídeos cardiotóxicos, tais como Nerium oleander e Kalanchoe blossfeldiana. Recentemente, várias metodologias experimentais foram empregadas para evitar intoxicações por plantas que contêm monofluoroacetato de sódio. Estas metodologias incluem a indução de aversão condicionada utilizando cloreto de lítio, a utilização de doses repetidas não tóxicas de folhas para induzir resistência, o uso de acetamida para prevenir as intoxicações e a inoculação intraruminal de bactérias degradantes de monofluoroacetato de sódio.

TERMOS DE INDEXAÇÃO: Plantas tóxicas, glicosídeos cardiotóxicos, monofluoroacetato de sódio, intoxicação por plantas, ruminantes, toxicoses.

\section{INTRODUÇÃO}

No Brasil, plantas pertencentes às famílias Rubiaceae, Malpighiaceae, Bignoniaceae, Fabaceae, Apocynaceae e Crassulaceae constituem um grupo muito importante de plantas tóxicas para animais de produção por afetarem o funcionamento do coração (Tokarnia et al. 2012). De acordo com dados dos laboratórios de diagnóstico de diferentes regiões do país, estima-se que esse grupo de plantas seja responsável pela morte de cerca de 500 mil bovinos por ano. Considerando o preço médio de US $\$ 200,00$ por animal, as perdas anuais podem ultrapassar a cifra de um milhão de dólares (Riet-Correa \& Medeiros 2001, Tokarnia et al. 2012). Considerando a importância econômica desse grupo de plantas no Brasil, este trabalho tem como objetivo atualizar os dados sobre os principais aspectos epidemiológicos, clínicos, patológicos e formas de controle das intoxicações por plantas que afetam o funcionamento do coração de ruminantes.

\section{REVISÃO DE LITERATURA}

\section{Plantas que provocam mortes súbitas associadas ao exercício}

De uma maneira geral, as plantas que provocam mortes súbitas associadas ao exercício contêm concentrações elevadas de monofluoroacetato de sódio (MFA) e por esse motivo o quadro clínico-patológico apresenta evolução superaguda, sem que se observem lesões cardíacas significantes. Esse grupo de plantas é atualmente representado por 22 espécies pertencentes às três famílias: Rubiaceae (Palicourea e Psychotria), Malpighiaceae (Amorimia e Niedenzuella) e Bignoniaceae (Tanaecium e Fridericia).

\section{Plantas dos gêneros Palicourea e Psychotria}

No gênero Palicourea, são importantes as intoxicações por P. marcgravii, $P$. aeneofusca, $P$. juruana e $P$. grandiflora (Tokarnia et al. 2012). Porém, em pesquisas recentes detectou-se monofluoroacetato de sódio em mais oito espécies: P. amapaensis, $P$. longiflora, $P$. barraensis (anteriormente $P$. aff. longiflora), $P$. macarthurorum, $P$. nigricans, $P$. vacillans e $P$. aff. juruana. No gênero Psychotria, só existem relatos de intoxicação por Psychotria hoffmannseggiana (Cook et al. 2014, Pedroza 2015, Carvalho et al. 2016).

Desse grupo, P. marcgravii é a planta tóxica mais importante devido à sua vasta distribuição no Brasil, ocorrendo nos domínios amazônia, caatinga, cerrado e mata atlântica. Provoca altos índices de mortalidade devido à sua boa palatabilidade, como por exemplo, na região amazônica, onde é responsável por $80 \%$ de todas as mortes relacionadas às intoxicações por plantas em bovinos (Tokarnia et al. 2012). Palicourea aeneofusca é importante nos estados de Pernambuco, Paraíba, leste da Bahia e Alagoas, onde ocorre em áreas de mata atlântica do litoral e zona da mata e no agreste, em matas de brejo de altitude (Vasconcelos et al. 2008a, Brito et al. 2016). A intoxicação natural é frequente em bovinos e ocasionalmente é relatada em pequenos ruminantes (Albuquerque et al. 2013, Brito et al. 2016, Oliveira-Neto et al. 2017).

Várias espécies que contêm MFA têm sua ocorrência registrada na região amazônica e podem ser encontradas em florestas de terra firme, florestas e campos de várzea, como P. grandiflora (Acre, Amazonas, Pará, Rondônia e Mato Grosso), P. amapaensis (Amazonas, Amapá e Pará), P. macarthurorum (Amazonas), P. longiflora (Amazonas, Pará e Rondônia), P. nigricans (Acre e Amazonas), $P$. barraensis (Amazonas, Roraima e Rondônia) e $P$. aff. juruana (Maranhão e Tocantins). Palicourea vacillans encontra-se distribuída em áreas de cerrado nos estados de Minas Gerais e Bahia (Cook et al. 2014, Taylor 2015a, 2015b, Carvalho et al. 2016). Das espécies acima citadas existem casos registrados de surtos de morte súbita em bovinos associados às intoxicações por $P$. grandiflora, $P$. barraensis e $P$. longiflora. Experimentalmente, $P$. longiflora e P. barraensis também causam intoxicação em coelhos (Cook et al. 2014, Carvalho et al. 2016).

A classificação do gênero Psychotria é complexa e não está totalmente resolvida, posto que algumas espécies atualmente classificadas como Psychotria estão mais intimamente relacionadas com o gênero Palicourea (Taylor 1996, Taylor et al. 2010, Taylor \& Gereau 2013). Desse gênero, Psychotria hoffmannseggiana é provavelmente importante por ser endêmica em todo o país. Psychotria hoffmannseggiana é um arbusto que mede de 0,5 a $2 \mathrm{~m}$ e pode ser encontrado na mata atlântica, em matas mesófilas, semidecíduas, restingas, matas ciliares e no cerrado (Taylor 2007). No Brasil, há somente dois registros de intoxicação por P. hoffmannseggiana em bovinos, nos estados de Minas Gerais e Rondônia (Schons 2011, Pedroza 2015). Nessas regiões, produtores rurais e médicos veterinários relatam há muito tempo surtos de mortes súbitas em bovinos. No Vale do Paraíba, Palicourea barbiflora (sinônimo de P. hoffmannseggiana) foi relatada como responsável por surtos de mortes súbitas em bovinos em décadas passadas e sua toxicidade foi confirmada experimentalmente em bovinos (Camargo 1962). A concentração de MFA em P. hoffmannseggiana ainda não foi determinada (Pedroza 2015). 
A concentração de MFA difere entre as espécies do gênero Palicourea. Em um estudo mais recente com métodos mais precisos (Lee et al. 2012), as concentrações foram significativamente maiores que as relatadas em estudos anteriores, cujos valores eram de $0.00054 \%$ de MFA (Krebs et al. 1994, Tokarnia et al. 2012). No estudo publicado por Cook et al. (2014) as concentrações de MFA nas folhas maduras de $P$. marcgravii foram estabelecidas em $0,24 \pm 0,10 \%$ e $0,88 \pm 0,08 \%$ nas folhas jovens e em desenvolvimento. Já nas folhas de $P$. aeneofusca, a concentração foi de $0,09 \pm 0,05 \%$ (Lee et al. 2012). Nas folhas de P. longiflora a concentração variou de $0.006 \%$ a $0.16 \%$ e nas folhas de $P$. barraensis a concentração foi de $0.01 \%$ (Carvalho et al. 2016).

As concentrações de MFA nas folhas de P. juruana, P. grandiflora, P. amapaensis, P. macarthurorum, P. nigricans, $P$. vacillans e $P$. aff. juruana ainda não foram determinadas. Sugeriu-se, porém, que devem ser similares às encontradas em marcgravii, P. aeneofusca, P. barraensis e P. longiflora (Cook et al. 2014, Carvalho et al. 2016). A dose letal das folhas frescas de P. marcgravii para bovinos foi estabelecida em $0,6 \mathrm{~g} / \mathrm{kg}$, sendo similar a dose letal para pequenos ruminantes (Tokarnia et al. 2012). A menor dose de folhas frescas de P. aeneofusca capaz de matar um bovino foi de $0,75 \mathrm{~g} / \mathrm{kg}$ (Tokarnia et al. 1983). A dose letal das folhas de P. juruana foi estabelecida em $2,0 \mathrm{~g} / \mathrm{kg}$ e de . grandiflora entre 1,0 e $2,0 \mathrm{~g} / \mathrm{kg}$ (Peixoto et al. 2011). Para coelhos, a dose letal das folhas de P. barraensis e P. longiflora foi de $2 \mathrm{~g} / \mathrm{kg}$ (Carvalho et al. 2016). Assumindo-se que as concentrações de MFA são similares em Palicourea spp., é presumível que as doses letais e o quadro clínico-patológico sejam similares. Portanto, são potencialmente tóxicas se as folhas e frutos forem consumidos por animais de fazenda.

\section{Plantas dos gêneros Amorimia e Niedenzuella}

Dentre as Malpighiaceae, são importantes as intoxicações por A. amazonica, A. exotropica, A. pubiflora, A. rigida e A. septentrionalis (Duarte et al. 2013). No gênero Niedenzuella são importantes as intoxicações por N. stannea, N. multiglandulosa e N. acutifolia. É importante ressaltar que várias espécies anteriormente classificadas como Mascagnia foram reclassificadadas como Amorimia (Anderson 2006) e que várias espécies anteriormente classificadas como Tetrapterys foram reclassificadas como Niedenzuella (Davis \& Anderson 2010). As informações sobre as intoxicações por $N$. multiglandulosa e $N$. acutifolia (anteriormente denominadas Tetrapterys multiglandulosa e Tetrapterys acutifolia) estão apresentadas entre as plantas que provocam fibrose cardíaca, pois, apesar de conterem MFA como princípio tóxico, raramente provocam doença com evolução superaguda. Além disso, provocam aborto e sinais clínicos e lesões relacionadas ao sistema nervoso central que não são descritas nas plantas que provocam mortes súbitas associadas ao exercício.

Amorimia amazonica ocorre nas florestas de Igapó da região amazônica nos estados do Acre, Amazonas e Rondônia (Schons et al. 2011, Duarte et al. 2013, Mamede 2015). Nos estados de Rondônia e Mato Grosso é responsável por surtos de mortes súbitas associadas ao exercício em bovinos e ovinos no Vale do Anari. As intoxicações ocorrem durante todo o ano, porém são mais frequentes no início do período chuvoso quando há pouca disponibilidade de forragens e A. amazonica está brotando (Schons et al. 2011).
Amorimia exotropica ocorre em locais sombreados na mata atlântica do Sudeste (São Paulo) e Sul (Paraná, Rio Grande do Sul e Santa Catarina). O primeiro surto de intoxicação em bovinos foi descrito no Estado de Santa Catarina (Gava et al. 1998) e mais recentemente nas regiões metropolitanas de Porto Alegre, Serra Gaúcha e microrregião de Vacaria, Rio Grande do Sul (Pavarini et al. 2011, Bandinelli et al. 2014). As intoxicações ocorrem durante todo o ano, porém, com maior concentração de casos entre os meses de maio e agosto, período em que há escassez de alimentos no Sul do Brasil. Desta forma, os bovinos podem invadir áreas de matas ou capões à procura de alimento. Bovinos também podem se intoxicar quando adentram nas matas à procura de abrigo das chuvas e ventos frios que também se concentram nessa época (Pavarini et al. 2011).

Amorimia pubiflora tem ocorrência registrada no cerrado da região Centro-Oeste (Goiás, Mato Grosso do Sul e Mato Grosso) e na mata atlântica da região Sudeste (Minas Gerais e São Paulo). Porém, surtos de intoxicação em bovinos só foram registrados em Mato Grosso e Mato Grosso do Sul. Nas regiões de sua ocorrência, $A$. pubiflora é uma das plantas tóxicas mais importantes, chegando a ser um fator limitante para expansão pecuária devido à ocorrência de mortes súbitas, com registros de mortalidade próximos a 50\% (Becker et al. 2013). Em Mato Grosso do Sul, $A$. pubiflora foi responsável por vários surtos de mortes súbitas em diferentes meses do ano, mas os casos predominaram no período chuvoso com morbidade de até 3,5\% e a taxa de letalidade de 100\% (Lemos et al. 2011). Em Mato Grosso, as mortes de bovinos ocorrem mesmo com disponibilidade de forragem, no entanto é frequentemente relatado que as mortes se concentram no final do período da seca e início do período chuvoso (Becker et al. 2013).

Amorimia rigida têm sua ocorrência registrada na Bahia, Espírito Santo, Minas Gerais e Rio de Janeiro; seu habitat são as áreas de caatinga e da mata atlântica (Mamede 2015) e, segundo Tokarnia et al. (1985) ocorre nos lugares mais baixos das pastagens, pés-de-serra e beiras de rios. É possível que A. rigida ocorra em outros estados do Nordeste, porém, nos surtos mais recentes de intoxicações relatados nessa região, a espécie responsável foi $A$. septentrionalis (Vasconcelos et al. 2008 b, Albuquerque et al. 2014). Intoxicações por A. rigida ocorrem principalmente na espécie bovina (Tokarnia et al. 1961, 1994, Medeiros et al. 2002, Da Silva et al. 2006, Vasconcelos et al. 2008b). Em caprinos e ovinos são menos frequentes (Oliveira et al. 1978, Pacífico da Silva et al. 2008, Lago et al. 2009). A intoxicação por A. rigida ocorre principalmente no início do período chuvoso quando está em brotação e as outras plantas ainda não cresceram. A planta também pode brotar após queimadas, período este também considerado como o de maior número de casos de intoxicações (Borboleta 2010, Tokarnia et al. 2012).

Amorimia septentrionalis ocorre sobre afloramentos rochosos da caatinga nos estados de Alagoas, Ceará, Paraíba, Pernambuco e Rio Grande do Norte (Mamede 2015). As intoxicações têm sido descritas principalmente no estado da Paraíba e em Pernambuco. É uma das principais plantas tóxicas para bovinos da região do Médio Capibaribe (Albuquerque et al. 2014). A doença ocorre principalmente no início do período chuvoso, quando a planta brota antes que outras forrageiras ou após o final desse período, quando algumas forrageiras secam e A. septentrionalis permanece verde (Vasconcelos et al. 2008b). 
Niedenzuella stannea tem ocorrência confirmada nas regiões Centro-Oeste (Mato Grosso e Mato Grosso do Sul) e Norte (Acre, Amazonas, Amapá, Pará, Rondônia, Tocantins) e ocorre tanto na floresta amazônica quanto no pantanal. Até o momento, só existem históricos de intoxicações em bovinos na região do Araguaia, Mato Grosso. Os surtos ocorrem principalmente durante a estação seca, entre junho e agosto, quando a planta está em brotação. A toxicidade da planta foi demonstrada em bovinos e ovinos pela administração de folhas jovens (Caldeira et al. 2016, Arruda et al. 2017). A evolução da intoxicação é aguda ou hiperaguda, dependendo da quantidade de planta ingerida. Experimentalmente em bovinos, doses de $15 \mathrm{~g} / \mathrm{kg}$ produziram sinais clínicos seguidos de recuperação. A menor dose que provocou a morte de bovinos foi $20 \mathrm{~g} / \mathrm{kg}$ (Arruda et al. 2017).

A concentração de MFA em A. amazonica, A. exotropica, A. pubiflora, $A$. rigida e $A$. septentrionalis foram determinadas em amostras de herbário, com concentrações nas folhas de 0,0007\%, 0,02\%, 0,06\%, 0.002\% e 0.002\% respectivamente. Porém, nessas espécies sabe-se que existe variação da quantidade de MFA tanto nas folhas quanto nas flores, caules e sementes (Lee et al. 2012). Experimentalmente, sinais clínicos de intoxicação foram observados com doses únicas entre 3,0 e 6,0g/Kg das folhas de A. amazonica. Doses únicas entre 7,5-10g/ kg de folhas frescas de $A$. exotropica causaram intoxicação e morte de bovinos (Gava et al. 1998) e doses entre $5-20 \mathrm{~g} / \mathrm{kg}$ das folhas de A. pubiflora também foram capazes de causar sinais clínicos de intoxicação em bovinos. Devido à significante variação da toxidez de $A$. rigida para bovinos, não se determinou a dose letal para essa espécie (Tokarnia et al. 2012). Em coelhos, a dose letal foi de $4 \mathrm{~g} / \mathrm{kg}$ das folhas dessecadas e $0,25 \mathrm{~g} / \mathrm{kg}$ dos frutos dessecados (Tokarnia et al. 1987). Na reprodução experimental da intoxicação por $A$. septentrionalis em caprinos e ovinos, doses únicas de 10 e $20 \mathrm{~g} / \mathrm{kg}$ foram letais. Já animais que receberam a dose de $5 \mathrm{~g} / \mathrm{kg}$ apresentaram sinais discretos e se recuperaram da intoxicação (Vasconcelos et al. 2008b).

As concentrações de MFA foram altamente variáveis entre as diferentes partes de $N$. stannea. As concentrações foram maiores nas sementes $(0,06 \%)$, seguidas de frutos $(0,0008$ a $0,02 \%)$, flores $(0,0003$ a $0,006 \%)$ e folhas $(0,0003 \%)$. Experimentalmente em bovinos, doses de $15 \mathrm{~g} / \mathrm{kg}$ provocaram apenas sinais clínicos com consequente recuperação dos animais. Doses entre $20-30 \mathrm{~g} / \mathrm{kg}$ provocaram sinais clínicos e morte (Arruda et al. 2017).

\section{Plantas dos gêneros Tanaecium e Fridericia}

Dentre as Bignoniaceae, são importantes as intoxicações por Tanaecium bilabiatum (anteriormente denominada Arrabidea bilabiata) e Fridericia elegans (anteriormente denominada Pseudocalymma elegans) (Lima et al. 2016, Santos-Barbosa et al. 2017). T. bilabiatum tem ampla ocorrência registrada no país e pode ser encontrada na floresta amazônica, caatinga, cerrado, mata atlântica, pampa e pantanal (Lohmann \& Taylor 2014). Na Bacia Amazônica, essa planta é responsável por numerosas mortes de bovinos que ocorrem em extensas regiões de várzea e, segundo Tokarnia et al. (2012), depois de P. marcgravii, é a principal planta tóxica da região Amazônica para bovinos.

No estado de Roraima, surtos de intoxicações em bovinos com quadro clínico de mortes súbitas são atribuídos à Fridericia japurensis (anteriormente denominada Arrabidea japurensis)
(Tokarnia \& Döbereiner 1981). No entanto, recentemente descobriu-se que a identificação de F. japurensis a partir de amostras de plantas coletadas em fazendas dessa região estava incorreta. Também se verificou que não existe MFA em F. japurensis, tanto em amostras coletadas em fazendas com casos confirmados de mortes súbitas, quanto em amostras de herbário. Na verdade, a planta responsável por surtos de mortes súbitas de bovinos no Estado de Roraima é também T. bilabiatum (Lima et al. 2016).

Fridericia elegans tem seu registro confirmado apenas no Rio de Janeiro, onde cresce no bioma Mata atlântica, especificamente nas costas dos morros de florestas caducifólia e ombrifólia (Lohmann 2015). Sabe-se de sua ocorrência nos municípios de São Miguel Pereira, Pacarambi, Saquarema, Rio Bonito e Campo Grande (Tokarnia et al. 2012). Sob condições naturais, a intoxicação por esta planta tem sido observada em bovinos (Helayel et al. 2009) e há histórico de intoxicação em equinos. Experimentalmente podem ser intoxicados bovinos, equinos, coelhos, caprinos e ovinos (Tokarnia et al. 1993, Consorte et al. 1994, Helayel et al. 2009, Tokarnia et al. 2012).

A concentração de MFA nas folhas de T. bilabiatum variou significantemente de acordo com os locais de coleta, sendo determinada entre $0,0001 \%$ e $0,039 \%$ (Lima et al. 2016) e experimentalmente em coelhos, a menor dose que causou morte foi de $0,5 \mathrm{~g} / \mathrm{kg}$ com a brotação coletada na época da seca. Já com as folhas maduras, a menor dose que causou a morte dos coelhos foi de 4,0g/kg (Jabour et al. 2006). Para bovinos, $1,25 \mathrm{~g} / \mathrm{kg}$ das folhas frescas causaram sinais graves de intoxicação, enquanto $2,5 \mathrm{~g} / \mathrm{kg}$ provocaram a morte (Döbereiner et al. 1983). A concentração de MFA em F. elegans não foi determinada. A administração, por via oral, de doses entre 0,5 e $1 \mathrm{~g} / \mathrm{kg}$ da brotação da planta causou sinais clínicos de intoxicação e morte de bovinos, enquanto a dose de $0,25 \mathrm{~g} / \mathrm{kg}$ foi capaz de causar apenas sinais clínicos, mas não levou à morte (Helayel et al. 2009).

\section{Sinais clínicos, lesões macro e microscópicas associadas às intoxicações por plantas que contém monofluoroacetato de sódio (MFA)}

O MFA é altamente tóxico para todas as espécies de mamíferos, incluindo humanos. No entanto, os efeitos tóxicos são muito variáveis em função da espécie intoxicada, da sensibilidade individual e dose ingerida, McIlroy 1981, Tokarnia et al. 2012). Porém, em ruminantes o quadro clínico consiste principalmente em intoxicações com evolução hiperaguda. 0 início dos sinais clínicos se dá poucas horas após a ingestão da dose tóxica e o exercício físico ou movimentação pode precipitar ou causar os sinais clínicos (entre 4-30 horas) (Tokarnia et al. 2012). Os sinais clínicos da intoxicação em bovinos, búfalos, caprinos e ovinos consistem em anorexia, apatia, letargia, micções frequentes, decúbito esternal prolongado, pulso venoso positivo, taquicardia, taquipneia, dispneia, instabilidade, tremores musculares, quedas e, durante a fase agônica, apresentam decúbito lateral, movimentos de pedalagem, opistótono, vocalizações e morte. Também podem estar presentes sinais clínicos decorrentes do decúbito prolongado, tais como diminuição dos movimentos ruminais, algumas vezes atonia, leve timpanismo e fezes ressecadas (Helayel et al. 2009). Esses sinais clínicos podem variar nas intoxicações, tendo em vista que a quantidade de MFA é significantemente variável nas espécies descritas neste trabalho. 
Os achados de necropsia são ausentes na maioria dos casos de intoxicação por plantas que contêm MFA em ruminantes. Porém, lesões inespecíficas tais como hemorragias petequiais no epicárdio, endocárdio e músculos papilares podem ser observadas e são geralmente decorrentes da fase agônica de morte. Outros achados consistem em hidropericárdio, edema e congestão pulmonar, palidez dos rins, congestão de grandes vasos e da mucosa do intestino delgado. Os achados histológicos são escassos ou, quando presentes, são condizentes com alterações degenerativas ou circulatórias, tais como degeneração hidrópico-vacuolar das células epiteliais renais, principalmente dos túbulos contorcidos distais com evidente picnose nuclear. Na intoxicação por P. aeneofusca descreveramse lesões inespecíficas no coração, tais como áreas difusas de hemorragia, edema das células de fibras de Purkinje e necrose coagulativa de fibras miocárdicas, com fibras em cariólise ou picnose, aumento da eosinofilia citoplasmática e perda das estriações transversais. Nos miocardiócitos podem ser observados vacúolos intranucleares discretos com marginalização da cromatina e nos espaços intersticiais pode haver edema discreto, depósitos de fibrina e infiltração de células mononucleares (Brito et al. 2016, Arruda et al. 2017). Possivelmente essas lesões também podem ser encontradas em outras intoxicações por plantas que contêm MFA.

Na intoxicação por Amorimia spp. em ruminantes, o quadro clínico e as lesões macro e microscópicas também são típicas de insuficiência cardíaca aguda. Entretanto, adicionalmente, podem incluir coágulos no interior do ventrículo esquerdo, congestão hepática e do trato gastrintestinal (Tokarnia et al. 1961, 1994, Paraguassu 1983, Pacífico da Silva et al. 2008, Vasconcelos et al. 2008b, Pavarini et al. 2011). Como as concentrações de MFA são menores em Amorimia spp., a letalidade é relativamente mais baixa do que a letalidade de Palicourea spp. (Medeiros et al. 2002). Em alguns casos, como nas intoxicações por A. exotropica, A. amazonica e A. septentrionalis, a evolução pode ser subaguda e, dessa forma, o coração pode exibir aspecto globular com áreas esbranquiçadas no miocárdio, além de áreas focais vermelhas entremeadas por áreas brancacentas na musculatura papilar esquerda (Soares et al. 2011, Bandinelli et al. 2014, Brito et al. 2016). Sendo assim, pode haver necrose de coagulação com infiltrado inflamatório composto por macrófagos, linfócitos, neutrófilos degenerados, além de tecido conjuntivo (fibrose) e debris celulares (Bandinelli et al. 2014, Brito et al. 2016).

\section{Diagnóstico, tratamento e medidas preventivas ou de controle}

Para o diagnóstico é fundamental o conhecimento das plantas que contém MFA que ocorrem em cada região. A presença dessas plantas associadas ao histórico de que os ruminantes morrem ou adoecem ao serem movimentados sugerem o diagnóstico de intoxicação. A lesão microscópica dos rins, quando presente, é de grande valor diagnóstico. A presença de fibrose cardíaca também é de valor diagnóstico para os casos de intoxicação subaguda por Amorimia spp. (Tokarnia et al. 2012). Recentemente foi demonstrado que o MFA pode ser detectado no soro sanguíneo de animais que foram intoxicados por plantas que contêm esse composto, contudo, esse método ainda não está disponível na rotina médico-veterinária (Santos-Barbosa et al. 2017).
Não existe tratamento para as intoxicações por plantas que contém MFA. O controle das intoxicações é difícil, especialmente em fazendas muito extensas. Nesses casos, a utilização de cercas ou a remoção manual das plantas não são medidas eficazes. A utilização de herbicidas é dispendiosa e só é eficaz em pequenas áreas, as quais também podem ser cercadas (Riet-Correa \& Medeiros 2001).

A utilização de doses entre 2,0 e 3,0g/kg de acetamida pode ser eficaz para a prevenção da intoxicação, uma vez que esse composto apresenta efeito protetor contra o MFA. Em um estudo realizado no Brasil, o tratamento prévio com acetamida evitou o aparecimento de sinais clínicos e o óbito de ovinos e caprinos intoxicados por F. elegans (Helayel et al. 2011). No entanto, a eficácia da acetamida depende de diversos fatores, como a toxidez da planta em questão, tempo decorrido entre a intoxicação e o fornecimento do antídoto e doses de acetamida administradas (Egyed \& Schultz 1986).

Em bovinos foi induzida experimentalmente aversão alimentar condicionada em condições de campo, administrando-se cloreto de lítio após a ingestão de P. aeneofusca. Nessa espécie, o efeito aversivo perdurou por 12 meses, mostrando-se um método eficaz (Brito et al. 2016). Em ovinos (Pacífico da Silva \& Soto-Blanco 2010) e caprinos (Barbosa et al. 2008) a mesma técnica foi utilizada para prevenir a intoxicação por A. septentrionalis com resultados satisfatórios em condições experimentais. Também foi comprovado que a administração de doses não tóxicas repetidas de folhas de $A$. septentrionalis induz resistência à intoxicação por essa planta e que essa resistência pode ser transmitida de animais resistentes para animais susceptíveis pela transfaunação de líquido ruminal (Duarte 2012). A mesma técnica foi utilizada com sucesso contra a intoxicação por A. pubiflora (Becker et al. 2016).

Em outro estudo, a inoculação intraruminal de bactérias degradadoras de MFA foi recentemente utilizada com sucesso para a prevenção da intoxicação por A. septentrionalis em que a administração intraruminal contínua de Ralstonia sp. e Burkholderia sp. proporcionou proteção completa em caprinos (Da Silva et al. 2016). Entretanto, esses métodos não estão disponíveis comercialmente. Em fazendas onde a intoxicação é frequente um método eficaz pode ser deixar os animais sem movimentação, em áreas em que não existem as plantas, por um período de 7 a 15 dias (Tokarnia et al. 2012).

\section{Plantas que provocam fibrose cardíaca}

Nesse grupo existem três espécies de plantas tóxicas: Niedenzuella multiglandulosa, anteriormente denominada Tetrapterys multiglandulosa e Niedenzuella acutifolia, anteriormente denominada Tetrapterys acutifolia (ambas da família Malpighiaceae) (Tokarnia et al. 1989, Riet-Correa et al. 2001, Anderson 2006, Carvalho et al. 2006, Caldas et al. 2011) e Ateleia glazioveana (da família Fabaceae) (Gava et al. 2001, Gava et al. 2003, Rissi et al. 2007). Por produzirem intoxicações bastante semelhantes em relação aos quadros clínicos e patológicos, serão apresentadas em conjunto. De uma forma geral, as intoxicações por esse grupo de plantas apresentam evolução subaguda à crônica e cursam com alterações degenerativas e fibrose cardíaca.

Niedenzuella multiglandulosa tem ocorrência registrada nos estados da Bahia, Goiás, Mato Grosso do Sul, Minas Gerais, Espírito Santo, Rio de Janeiro, São Paulo e Paraná. N. acutifolia tem ocorrência descrita no Acre, Amazonas, Mato Grosso, 
Goiás, Tocantins, Maranhão, Pernambuco, Bahia, Minas Gerais, Espírito Santo, Rio de Janeiro, São Paulo, Paraná e Santa Catarina, podendo ocorrer no cerrado, mata atlântica e floresta amazônica (Mamede 2015). Os casos de intoxicação por $N$. multiglandulosa foram registrados no Rio de janeiro, São Paulo e Mato Grosso do Sul. Os casos de intoxicação por $N$. acutifolia foram constatados em diversos municípios dos estados do Rio de Janeiro, São Paulo, Minas Gerais e Espírito Santo. As intoxicações ocorrem durante todo o ano e a morbidade varia de 6 a 28\%, com letalidade próxima a 100\% (Tokarnia et al. 1989, Riet-Correa et al. 2001).

As intoxicações naturais ocorrem somente em bovinos e, diferentemente das intoxicações por $N$. stannea, as intoxicações por N. multiglandulosa e N. acutifolia têm evolução subaguda à crônica (Carvalho et al. 2006). A doença foi reproduzida experimentalmente em bovinos por ingestão dos brotos frescos nas doses de $5 \mathrm{~g} / \mathrm{kg}$ durante 60 dias, $10 \mathrm{~g} / \mathrm{kg}$ durante 13 a 41 dias e $20 \mathrm{~g} / \mathrm{kg}$ durante 10 dias. Em um experimento com bovinos, doses únicas de $100 \mathrm{~g} / \mathrm{kg}$ não provocaram morte súbita. Constataram-se apenas sinais clínicos discretos (Tokarnia et al. 1989). Experimentalmente a intoxicação também foi reproduzida em ovinos (Riet-Correa et al. 2005, Cardinal et al. 2010).

Ateleia glazioveana, árvore com cerca de 5-15m de altura e $20-30 \mathrm{~cm}$ de diâmetro, ocorre na Mata Atlântica, nas florestas estacionais semideciduais e em florestas ombrófilas, principalmente nos Estados do Paraná, Rio Grande do Sul e Santa Catarina. Mas também possui ocorrência registrada nos Estados do Espírito Santo, Rio de Janeiro, São Paulo, Mato Grosso do Sul e Rio Grande do Norte (Mansano et al. 2015). Sob condições naturais, surtos de intoxicações foram descritos principalmente em bovinos no Oeste de Santa Catarina e Noroeste, Centro-Norte e Planalto Médio do Rio Grande do Sul (Gava et al. 2001, Rissi et al. 2007). Intoxicações em ovinos também podem ocorrer (Gava et al. 2003).

As intoxicações em bovinos geralmente estão associadas ao período de escassez de alimentos, principalmente quando ocorre seca no outono ou em invernos mais rigorosos. A maior maturação das folhas que ocorre no outono e a maior produção de sementes são fatores importantes para a ocorrência da doença, pois nesses casos a planta apresenta maior toxicidade (Gava et al. 2001). A intoxicação por A. glazioveana foi reproduzida experimentalmente em bovinos que consumiram entre 40 e $50 \mathrm{~g} / \mathrm{kg}$ das folhas verdes uma única vez. Lesões cardíacas crônicas foram reproduzidas experimentalmente em bovinos, com doses fracionadas de 2,5, 5,0 e 7,5g/kg por longo período e com dose inicial de $1 \mathrm{~g} / \mathrm{kg}$, acrescida de $1 \mathrm{~g} / \mathrm{kg} / \mathrm{dia}$ até atingir $15 \mathrm{~g} / \mathrm{kg}$, num total de $120 \mathrm{~g} / \mathrm{kg}$ (Gava et al. 2001). Em ovinos, doses fracionadas totalizando $60 \mathrm{ou} 80 \mathrm{~g} / \mathrm{kg}$ até $125 \mathrm{~g} / \mathrm{kg}$ das folhas de A. glazioveana causaram lesões cardíacas (Stigger et al. 2001, Raffi et al. 2006, Almeida et al. 2008).

O princípio tóxico de $A$. glazioveana é desconhecido. Porém, as lesões cardíacas observadas nessa intoxicação são semelhantes às observadas nas intoxicações por plantas da família Rubiaceae do Sul da África, que contêm como toxina uma substância do grupo das poliaminas denominada pavetamina, (García y Santos et al. 2004, Riet-Correa et al. 2005). Mais recentemente demonstrou-se que uma isoflavona extraída das folhas de A. glazioveana, denominada glaziovina A apresenta efeito citotóxico por inibir a polimerização de microtúbulos (Hayakawa et al. 2016). Todavia ainda não foram realizados experimentos para determinar se essa substância é responsável pelas lesões cardíacas presentes em bovinos intoxicados por A. glazioveana.

Niedenzuella multiglandulosa e $N$. acutifolia apresentam MFA como princípio ativo. Porém, as concentrações dessa toxina não foram publicadas, pois a detecção foi realizada em espécimes de herbário (Santos-Barbosa et al. 2017). Tendo em vista que as intoxicações apresentam evolução subaguda a crônica, é possível que a quantidade de MFA nessas espécies seja menor do que em Palicourea spp. ou que haja interação com outras substâncias tóxicas, possivelmente toxinas do grupo das poliaminas, similar ao que se sugere como princípio tóxico da A. glazioveana (García y Santos et al. 2004, Riet-Correa et al. 2005).

\section{Sinais clínicos, lesões macro e microscópicas associadas às intoxicações por plantas que provocam fibrose cardíaca}

Diferentemente das outras intoxicações por plantas que contém MFA, as intoxicações por N. multiglandulosa e N. acutifolia cursam sinais clínicos relacionados à insuficiência cardíaca congestiva. Além disso, causam sinais clínicos e lesões (edema intramielínico) relacionados ao sistema nervoso e são causa frequente de aborto e mortalidade neonatal com presença de lesões cardíacas e no sistema nervoso de fetos e neonatos (Riet-Correa et al. 2005, Carvalho et al. 2006).

Os principais sinais clínicos, porém, consistem em edemas subcutâneos nos locais de declive como edema de barbela e edema esternal ("peito inchado"), ingurgitamento e pulso venoso positivo da jugular, arritmia cardíaca, dispneia, letargia, anorexia, fraqueza, dificuldade de locomoção, tremores musculares e fezes ressequidas (Carvalho et al. 2006, Tokarnia et al. 2012). Nesses casos são observadas lesões cardíacas que consistem em áreas claras que podem ser vistas no epicárdio, manchas e feixes esbranquiçados, muito nítidos, ocupando áreas extensas do miocárdio, que às vezes está endurecido. Há alterações secundárias como edemas e derrames serosos em cavidades. 0 fígado apresenta, em quase todos os casos, alterações que consistem em aumento do padrão lobular e às vezes maior firmeza que o normal. Os principais achados histológicos restringem-se ao coração e fígado, sendo observados cardiomiócitos com aumento de volume e número de núcleos, picnose, citoplasma tumefeito e eosinofílico, áreas multifocais de fibrose, às vezes, circundada por extensas áreas focais de necrose massiva no coração e, no fígado, observa-se congestão, tumefação, vacuolização e lise de hepatócitos, fibrose periportal e centrolobular (Carvalho et al. 2009, Caldas et al. 2011, Tokarnia et al. 2012).

Bovinos intoxicados por A. glazioveana também podem apresentar três diferentes manifestações, com sinais cardíacos, neurológicos ou reprodutivos (García y Santos et al. 2004, Raffi et al. 2006). Esses sinais podem ocorrer separadamente ou em conjunto dentro de um mesmo surto (Gava et al. 2001, Gava \& Barros 2001). Os sinais clínicos mais comuns, para bovinos e ovinos intoxicados naturalmente ou experimentalmente incluem apatia, depressão, letargia, cegueira, andar lento e cambaleante, salivação, fezes secas, emagrecimento progressivo, decúbito frequente, podendo ocorrer mandíbula apoiada ao chão, ingurgitamento da jugular e leve edema na região esternal, taquicardia e taquipneia, relutância em mover-se, cabeça baixa, instabilidade dos membros pélvicos, movimentos de pedalagem e morte espontânea devido ao 
esforço (Gava et al. 2001, Stigger et al. 2001). O quadro clínico-patológico manifestado pelos bovinos intoxicados por A. glazioveana é semelhante ao produzido pela intoxicação por N. multiglandulosa e N. acutifolia. Essa planta, quando ingerida pelos bovinos em grande quantidade e por um curto período não causa lesões cardíacas significativas, mas, se ingerida em doses fracionadas por períodos mais longos, produz lesões cardíacas associadas à insuficiência cardíaca crônica com edemas de declive (Tokarnia et al. 1989, Gava et al. 2001, Carvalho et al. 2006). Alterações macro e microscópicas causadas pela intoxicação por A. glazioveana variam de acordo com a manifestação clínica (cardíaca, neurológica ou reprodutiva). Lesões macroscópicas incluem áreas esbranquiçadas no miocárdio e o fígado com aspecto de noz-moscada. As alterações histológicas mais citadas são tumefação e necrose de miofibras cardíacas, fibrose intersticial no miocárdio, e degeneração esponjosa no encéfalo (Gava et al. 2001, Stigger et al. 2001, Raffi et al. 2006).

\section{Diagnóstico, tratamento e medidas preventivas ou de controle}

O diagnóstico das intoxicações por plantas que provocam fibrose cardíaca deve ser baseado nos dados epidemiológicos, em que há presença das plantas e histórico de seu consumo associados à presença do quadro clínico bastante característico de insuficiência cardíaca, com evidente edema de barbela e da região esternal e ingurgitamento de jugulares em bovinos adultos. Em bovinos jovens esses sinais clínicos são menos característicos.

No diagnóstico diferencial deve se levar em consideração outras doenças cardíacas de bovinos como a pericardite traumática e as endocardites (Gava et al. 2001), que podem ser confundidas clinicamente com a intoxicação crônica por plantas que provocam fibrose cardíaca. Apesar disso, esses casos não ocorrem sob a forma de surtos, tendo lesões de necropsia características e que permitem o diagnóstico definitivo. Lesões cardíacas semelhantes também podem ser observadas nas intoxicações por antibióticos ionóforos e deficiência de selênio e também, apesar de serem raras, nas intoxicações pelas sementes de Senna occidentalis e Senna obtusifolia (Nogueira et al. 2009, Carmo et al. 2011, Tokarnia etal. 2012, Carvalho et al. 2014). Nessas condições a musculatura esquelética também é afetada, o que não ocorre nas intoxicações por A. glazioveana, N. multiglandulosa e N. acutifolia. Outra enfermidade que ocorre em bovinos adultos na região da Serra Geral de Santa Catarina, chamada de "doença do peito inchado", deve ser levada em consideração por apresentar quadro clínico similar às intoxicações por A. glazioveana, N. multiglandulosa e N. acutifolia. Porém, a lesão característica é uma intensa fibrose intersticial do miocárdio, não ocorrendo lesões degenerativo-necróticas.

Não se conhece tratamento eficaz para as intoxicações por plantas que provocam fibrose cardíaca. A profilaxia consiste na erradicação das plantas das áreas de pastagens. No caso de A. glazioveana a erradicação pode ser difícil porque o corte das árvores favorece a brotação, facilitando o acesso aos bovinos.

\section{Plantas que contêm glicosídeos cardiotóxicos}

Nesse grupo existem duas espécies de plantas tóxicas: Nerium oleander (Apocynaceae) (Leániz et al. 2013) e Kalanchoe blossfeldiana (Crassulaceae). Ambas as espécies são ornamentais e pouco importantes para animais de fazenda, tendo em vista que os surtos de intoxicação são menos frequentes e ocorrem por erros de manejo, geralmente quando as plantas são podadas e deixadas nas áreas de pastagem ou quando são, equivocadamente, oferecidas aos animais (Riet-Correa \& Méndez 2007).

Nerium oleander é uma planta arbustiva encontrada em todo o Brasil e que pode atingir 4 metros de altura (Zibbu \& Batra 2010, Koch et al. 2015). Surtos naturais de intoxicação foram diagnosticados apenas em bovinos nos estados da Paraíba, Rio Grande do Norte e Rio Grande do Sul (Soto-Blanco et al. 2006, Pedroso et al. 2009, Assis et al. 2010). A toxicidade de $N$. oleander se deve à elevada quantidade de cardenolídeos, sobretudo oleandrina, presente em todas as partes da planta, como folhas, ramas, flores, raízes e seiva, frescas ou secas (Zibbu \& Batra 2010). Os cardenolídeos inibem a enzima $\mathrm{Na}+/ \mathrm{K}+$ ATPase da membrana dos cardiomiócitos, promovendo redução do potássio e aumento do sódio intracelular, resultando no acúmulo de cálcio. Esses distúrbios eletrolíticos afetam a condutividade e contratibilidade do coração (Soto-Blanco et al. 2006). Estudos anteriores demonstraram que a média da concentração de oleandrina nas folhas de $N$. oleander é elevada, geralmente superior a 4,5 $\mathrm{mg} \mathrm{g}^{-1}$ (Soto-Blanco et al. 2006, Pedroza et al. 2015). Experimentalmente, em bovinos, as doses de 0,5 e 1,0g/kg de folhas de $N$. oleander desencadearam sinais clínicos severos e morte (Pedroso et al. 2009). De acordo com Soto-Blanco et al. (2006), a dose letal para esta espécie seria de $0,005 \%$ do peso corporal do animal de folhas secas. Em ovinos, a dose letal foi estimada entre 0,5 e $1 \mathrm{~g} / \mathrm{kg}$ de peso corporal de folhas verdes (Armién et al. 1994).

Kalanchoe blossfeldiana é uma espécie de suculenta com hábito perene e que mede aproximadamente $45 \mathrm{~cm}$ de altura. As folhas são de cor verde escura, opostas e têm aspecto elíptico e espatulado. Suas flores têm longa duração e as cores variam de branco a amarelo e tons de vermelho. Florescem no fim do inverno e início da primavera (Costa 2011). No Brasil só há um relato de intoxicação em bovinos no agreste de Pernambuco (Ribeiro et al. 2016). K. blossfeldiana contêm como princípio tóxico bufadienólidos, que também são potentes glicosídeos cardiotóxicos presentes em todas as partes da planta. Para bovinos, a dose letal das flores e folhas de Kalanchoe spp. foi estimada entre $7 \mathrm{~g} / \mathrm{kg}$ e $40 \mathrm{~g} / \mathrm{kg}$, respectivamente (Ribeiro et al. 2016).

\section{Sinais clínicos, lesões macro e microscópicas associadas às intoxicações por plantas que contêm glicosídeos cardiotóxicos}

Os sinais clínicos da intoxicação por $N$. oleander são observados entre 1 e 24 horas após a ingestão das folhas (Baskin et al. 2007) e consistem principalmente em andar cambaleante, polidipsia, desidratação, sialorreia, micção frequente, ranger de dentes, tremores musculares generalizados, aumento da frequência respiratória, dificuldade de locomoção, regurgitação de conteúdo ruminal, relutância em caminhar, queda brusca do animal ao chão, decúbito lateral, movimentos de pedalagem, vocalização, intensa taquicardia, mugidos, vômito, diarreia (às vezes sanguinolenta), ataxia, extremidades frias, dispneia, paralisia, coma e morte (Assis et al. 2010, Pedroso et al. 2009, Baskin et al. 2007). Há também efeitos diuréticos que estão ligados diretamente aos efeitos dos glicosídeos nos túbulos renais, especialmente nos estágios iniciais da intoxicação, 
resultando em elevação do fluxo sanguíneo renal e aumento da filtração glomerular e da diurese (Adams 1995). Em alguns casos também se pode notar hidrotórax, hidropericárdio e ascite.

As lesões macroscópicas da intoxicação por $N$. oleander são inespecíficas (Leániz et al. 2013) e consistem em congestão de mucosas (ocular, bucal e vulvar) e principalmente hemorragias. No coração essas hemorragias podem ser do tipo petequial até sufusões que podem ser notadas no epicardio, endocárdio e por vezes formam coágulos no átrio e ventrículo esquerdo. No miocárdio e septo interventricular pode haver ainda áreas levemente pálidas que correspondem à necrose. Os pulmões frequentemente encontram-se congestos e edemaciados. Hemorragias podem ser notadas também na pleura, tecidos subcutâneos, músculos intercostais, omento e serosa do rúmen. À superfície de corte, o fígado está frequentemente vermelho-escuro e os rins podem estar de coloração amarelopálida com petéquias na região medular (Pedroso et al. 2009, Assis et al. 2010, Di Paolo et al. 2010).

Ao exame microscópico nota-se achados em diferentes estruturas, como o miocárdio, com hemorragias subendoteliais locais e difusas, infiltrado de células mononucleares no endocárdio, nos músculos papilares dos ventrículos direito e esquerdo. Nas fibras musculares cardíacas pode haver picnose, aumento da eosinofilia, áreas extensas e multifocais de degeneração e necrose hialina e vacuolização e hemorragia subendocárdica (Pedroso et al. 2009, Di Paolo et al. 2010). No fígado pode haver áreas focais de degeneração e necrose de hepatócitos, com hemorragia, congestão difusa acentuada, células inflamatórias no parênquima e necrose individual de hepatócitos e, no baço, infiltrado linfohistiocitário na polpa branca, com macrófagos contendo hemossiderina (Pedroso et al. 2009, Assis et al. 2010, Di Paolo et al. 2010). 0 tecido renal pode apresentar congestão severa e extensa necrose nas células epiteliais dos túbulos distais e proximais, além de hemorragia e frequente pigmento de hemossiderina no citoplasma das células epiteliais dos túbulos renais (Aslani et al. 2007, Di Paolo et al. 2010).

Os sinais clínicos da intoxicação por K. blossfeldiana surgem poucas horas após a ingestão da planta e a evolução do quadro clínico, dependendo da quantidade de folhas ingeridas, dura em média de 4 a 5 dias. Os principais sinais clínicos incluem anorexia, inquietação, mucosas conjuntivais e vasos episclerais congestos, desidratação, muflo nasal ressecado, taquipneia, taquicardia, atonia ruminal, diarreia hemorrágica, prostração e decúbito lateral permanente seguido de morte (Ribeiro et al. 2016). À necropsia notam-se hemorragias petequiais e equimoses nas aurículas, epicárdio e músculos papilares. 0 fígado pode estar aumentado de volume, congesto e com a vesícula biliar distendida. 0 intestino delgado pode ter áreas de congestão severa e de hemorragias petequiais nas serosas e na mucosa pode haver hemorragia, por vezes contendo coágulos. As lesões microscópicas consistem em áreas extensas ou multifocais de degeneração e necrose de fibras musculares cardíacas. Na aurícula as principais lesões consistem em edema intersticial, infiltrado inflamatório, hemorragia e necrose de coagulação. Áreas focais de degeneração e necrose de hepatócitos também são constantemente observadas. Na submucosa do intestino delgado pode haver edema, presença de infiltrado inflamatório mononuclear e macrófagos contendo hemossiderina; já na mucosa são frequentes as áreas de hemorragia, diminuição da altura das vilosidades e necrose do epitélio, ocasionalmente associada à presença de infiltrado inflamatório de polimorfonucleares (Ribeiro et al. 2016).

\section{Diagnóstico, tratamento e medidas preventivas ou de controle}

0 diagnóstico de intoxicação por $N$. oleander e K. blossfeldiana deve ser baseado no histórico de consumo das plantas pelos animais, na epidemiologia, sinais clínicos, lesões macroscópicas e achados histológicos compatíveis com intoxicações por plantas que contém glicosídeos cardiotóxicos. No diagnóstico diferencial devem ser levadas em consideração as intoxicações por antibióticos ionóforos e as por plantas que contêm MFA (Tokarnia et al. 2012). Não existe antídoto para a intoxicação por $N$. oleander e K. blossfeldiana em bovinos. 0 tratamento deve ser sintomático e de suporte (Smith 2004).

\section{REFERÊNCIAS}

Adams H.R. 1995. Digitalis and vasodilator drugs, p.455-463. In: Adams H.R. (Ed.), Veterinary Pharmacology and Therapeutics. 7th ed. Iowa State University Press, Ames.

Albuquerque S.S., Brito L.B., Tavares R.M., Rocha B.P., Andrade M.L. \& Mendonça F.S. 2013. Mortes súbitas em caprinos associadas à intoxicação por Palicourea aeneofusca (Rubiacea) no Agreste de Pernambuco. Arch. Vet. Sci. 18(3):364-366.

Albuquerque S.S.C., Rocha B.P., Almeida V.M., Oliveira J.S., Riet-Correa F., Lee S.T., Evêncio Neto J. \& Mendonça F.S. 2014. Cardiac fibrosis associated to the poisoning by Amorimia septentrionalis in cattle. Pesq. Vet. Bras. 34(5):433-437. <http://dx.doi.org/10.1590/S0100-736X2014000500008>

Almeida M.B., Priebe A.P.S., Riet-Correa B., Riet-Correa G., Fiss L., Raffi M.B. \& Schild A.L. 2008. Evolução e Reversibilidade das Lesões Neurológicas e Cardíacas em Ovinos Intoxicados Experimentalmente por Ateleia glazioviana e Tetrapterys multiglandulosa. Pesq. Vet. Bras. 28(3):129-134. <http:// dx.doi.org/10.1590/S0100-736X2008000300001>

Anderson W.R. 2006. Eight segregates from the neotropical genus Mascagnia (Malpighiaceae). Novon, J. Bot. Nomenclature 16(2):168-204. <http:// dx.doi.org/10.3417/1055-3177(2006)16[168:ESFTNG]2.0.C0;2>

Armién A.G., Peixoto P.V., Barbosa J.D. \& Tokarnia C.H. 1994. Intoxicação experimental por Nerium oleander (Apocinaceae) em ovinos. Pesq. Vet. Bras. 14:85-93.

Arruda F.P.D., Caldeira F.H.B., Ducatti K.R., Bezerra K.S., Marcolongo-Pereira C., Lee S.T., Cook D., Riet-Correa F. \& Colodel E.M. 2017. Experimental poisoning by Niedenzuella stannea in cattle and corresponding detection of monofluoroacetate. Ciência Rural 47(3):1-6. <http://dx.doi.org/10.1590/01038478cr20160761>

Aslani M.R., Maleki M., Mohri M., Sharifi K., Najjar-Nezhad V. \& Afshari E. 2007. Castor bean (Ricinus communis) toxicosis in a sheep flock. Toxicon 49(3):400-406. <http://dx.doi.org/10.1016/j.toxicon.2006.10.010> $<$ PMid:17157890>

Assis T.S., Medeiros R.M.T., Riet-Correa F., Galiza G.J.N., Dantas A.F.M. \& Oliveira D.M. 2010. Intoxicações por plantas diagnosticadas em ruminantes e equinos e estimativa das perdas econômicas na Paraíba. Pesq. Vet. Bras. 30(1):13-20. <http://dx.doi.org/10.1590/S0100-736X2010000100003>

Bandinelli M.B., Bassuino D.M., Fredo G., Mari C., Driemeier D., Sonne L. \& Pavarini S.P. 2014. Identificação e distribuição de lesões cardíacas em bovinos intoxicados por Amorimia exotropica. Pesq. Vet. Bras. 34(9):837844. <http://dx.doi.org/10.1590/S0100-736X2014000900006>

Barbosa R.R., Pacífico da Silva I. \& Soto-Blanco B. 2008. Development of conditioned taste aversion to Mascagnia rigida in goats. Pesq. Vet. Bras. 28(12):571-574.<http://dx.doi.org/10.1590/S0100-736X2008001200001> 
Baskin S.I., Czerwinski S.E., Anderson J.B. \& Sebastian M.M. 2007. Cardiovascular toxicity, p.193-205. In: Gupta R.C. (Ed.), Veterinary Toxicology: basic and clinical principles. Elsevier, New York, NY. <http://dx.doi.org/10.1016/ B978-012370467-2/50110-3>.

Becker M., Caldeira F.H.B., Carneiro F.M., Oliveira L.P.D., Tokarnia C.H., RietCorrea F., Lee S.T. \& Colodel E.M. 2013. The importance of poisoning by Amorimia pubiflora (Malpighiaceae) in cattle in Mato Grosso: experimental reproduction of the poisoning in sheep and cattle. Pesq. Vet. Bras. 33(9):10491056. <http://dx.doi.org/10.1590/S0100-736X2013000900001>

Becker M., Carneiro F.M., Oliveira L.P.D., Silva M.I.V.D., Riet-Correa F., Lee S.T., Pescador C.A., Nakazato L. \& Colodel E.M. 2016. Induction and transfer of resistance to poisoning by Amorimia pubiflora in sheep whith non-toxic dosis of the plant and ruminal content. Ciência Rural 46(4):674-680. <http://dx.doi.org/10.1590/0103-8478cr20141484>

Borboleta L.R. 2010. Intoxicação experimental com extratos de Mascagnia rigida (A. Juss.) Griseb. (Malpighiaceae) em coelhos (Oryctolagus cuniculus): estudos clínico, laboratorial e anatomopatológico. Dissertação de Mestrado em Ciência Animal, Curso de Pós-graduação em Ciência Animal, Universidade Federal de Minas Gerais, Belo Horizonte. 142p.

Brito L.B.D., Albuquerque R.F., Rocha B.P., Albuquerque S.S., Lee S.T., Medeiros R.M.T., Riet-Correa F. \& Mendonça F.D.S. 2016. Spontaneous and experimental poisoning of cattle by Palicourea aeneofusca in the region of Pernambuco and induction of conditioned food aversion. Ciência Rural 46(1):138-143. <http://dx.doi.org/10.1590/0103-8478cr20150079>

Caldas S.A., Peixoto T.C., Nogueira V.A., França T.N., Tokarnia C.H. \& Peixoto P.V. 2011. Aborto em bovinos devido à intoxicação por Tetrapterys acutifolia (Malpighiaceae). Pesq. Vet. Bras. 31(9):737-746. <http://dx.doi. org/10.1590/S0100-736X2011000900003>

Caldeira F.B., Dias G.B., Arruda F.P., Lourenço F.M., Bezerra K.S., Riet-Correa F. \& Colodel E.M. 2016. Sudden death associated with Niedenzuella stannea (Malpighiaceae) in cattle in the State of Mato Grosso, Brazil: importance and epidemiological aspects. Pesq. Vet. Bras. 36:147-149.

Camargo W.A. 1962. Uma nova "erva-de-rato" tóxica para bovinos, Palicourea barbiflora: comparação com a Palicourea marcgravii var. pubescens e com Psychotria officinalis, Rubiaceae. Arqs Inst. Biológico, São Paulo, 29:1-11.

Cardinal S.G., Aniz A.C., Santos B.S., Carvalho N.M. \& Lemos R.A.A. 2010. Lesões perinatais em cordeiros induzidas pela administração de Tetrapterys multiglandulosa (Malpighiaceae) a ovelhas em diferentes estágios de gestação. Pesq. Vet. Bras. 30(1):73-78. <http://dx.doi.org/10.1590/ S0100-736X2010000100012>

Carmo P.M.S., Irigoyen L.F., Lucena R.B., Fighera R.A., Kommers G.D. \& Barros C.S.L. 2011. Spontaneous coffee senna poisoning in cattle: report on 16 outbreaks. Pesq. Vet. Bras. 31(2):139-146. <http://dx.doi.org/10.1590/ S0100-736X2011000200008>

Carvalho A.Q., Carvalho N.M., Vieira G.P., Santos A.C., Franco G.L., Pott A., Barros C.S.L. \& Lemos R.A.A. 2014. Intoxicação espontânea por Senna obtusifolia em bovinos no Pantanal Sul-Mato-Grossense. Pesq. Vet. Bras. 34(2):147-152. <http://dx.doi.org/10.1590/S0100-736X2014000200009>

Carvalho G.D., Nunes L.C., Bragança H.B.N. \& Porfírio L.C. 2009. Principais plantas tóxicas causadoras de morte súbita em bovinos no estado do Espírito Santo, Brasil. Arch. Zootec. 58:87-98.

Carvalho F.K.L., Cook D., Lee S.T., Taylor C.M., Soares Oliveira J.B. \& Riet-Correa F. 2016. Determination of toxicity in rabbits and corresponding detection of monofluoroacetate in four Palicourea (Rubiaceae) species from the Amazonas state, Brazil. Toxicon 109:42-44. <http://dx.doi.org/10.1016/j. toxicon.2015.11.009><PMid:26603601>

Carvalho N.M., Alonso L.A., Cunha T.G., Ravedutti J., Barros C.S.L. \& Lemos R.A.A. 2006. Intoxicação de Bovinos por Tetrapterys multiglandulosa (Malpighiaceae) em Mato Grosso do Sul. Pesq. Vet. Bras. 26(3):139-146. <http://dx.doi.org/10.1590/S0100-736X2006000300002>
Consorte L.B., Peixoto P.V. \& Tokarnia C.H. 1994. Intoxicação experimental por Pseudocalymma elegans (Bignoniaceae) em ovinos. Pesq. Vet. Bras. 14(4):123-133.

Cook D., Lee S.T., Taylor C.M., Bassüner B., Riet-Correa F., Pfister J.A. \& Gardner D.R. 2014. Detection of toxic monofluoroacetate in Palicourea species. Toxicon 80(15):9-16. <http://dx.doi.org/10.1016/j.toxicon.2013.12.003> $<$ PMid:24440601>

Costa T.N. 2011. Alterações hematológicas e bioquímicas séricas nas intoxicações de cães, gatos e ruminantes por plantas. Dissertação de Mestrado, Universidade Federal de Goiás, Goiânia. 30p.

Da Silva L.C., Pessoa D.A., Lopes J.R., de Albuquerque L.G., da Silva L.S., Garino Junior F. \& Riet-Correa F. 2016. Protection against Amorimia septentrionalis poisoning in goats by the continuous administration of sodium monofluoroacetate-degrading bacteria. Toxicon 111(1):65-68. <http://dx.doi.org/10.1016/j.toxicon.2015.12.016> <PMid:26747472>

Davis C.C. \& Anderson W.R. 2010. A complete generic phylogeny of Malpighiaceae inferred from nucleotide sequence data and morphology. Amer. J. Bot. 97(12):2031-2048.<http://dx.doi.org/10.3732/ajb.1000146> $<$ PMid:21616850>

Di Paolo L.A., Ancinas M.D., Tassara F., Peralta L.M. \& Hoyo L. 2010. Intoxicación natural con Nerium oleander en llamas (Lama glama) en un establecimiento de la provincia de Buenos Aires. Vet. Arg. 27(263):2-11.

Döbereiner J., Tokarnia C.H. \& Silva M.F. 1983. Intoxicação por Arrabidaea bilabiata em bovinos na Região Amazônica do Brasil. Pesq. Vet. Bras. 3(1):17-24.

Duarte A.L.L. 2012. Intoxicações por Amorimia spp. e Callaeum psilophyllum em ruminantes. Tese de Doutorado em Medicina Veterinária, Programa de Pós-Graduação em Medicina Veterinária, Universidade Federal de Campina Grande, PB. 68p.

Duarte A.L., Medeiros R.M.T. \& Riet-Corrêa F. 2013. Intoxicação por Amorimia spp. em ruminantes. Ciência Rural 43(7):1294-1301. <http://dx.doi. org/10.1590/S0103-84782013005000081>

Egyed M.N. \& Schultz R.A. 1986. The efficacy of acetamide for the treatment of experimental Dichapetalum cymosum (gifblaar) poisoning in sheep. Onderstepoort J. Vet. Res. 53(4):231-234. <PMid:3796950>

García y Santos M.C., Schild A.L., Barros S.S., Riet-Correa F., Elias F. \& Ramos A.T. 2004. Lesões perinatais em bovinos na intoxicação experimental por Ateleia glazioviana (Leg. Papilionoideae). Pesq. Vet. Bras. 24(8):178-184 . <http://dx.doi.org/10.1590/S0100-736X2004000400002>

Gava A. \& Barros C.S.L. 2001. Field Observations of Ateleia glazioviana Poisoning in Cattle in Southern Brazil. Vet. Hum. Toxicol. 43(1):37-41. <PMid:11205077>

Gava A., Barros C.S.L., Pilati C., Barros S.S. \& Mori A.M. 2001. Intoxicação por Ateleia glazioviana (Leg. Papilionoideae) em bovinos. Pesq. Vet. Bras. 21(2):49-59. <http://dx.doi.org/10.1590/S0100-736X2001000200003>

Gava D., Reis R.N., Rocha T.S., Pasquale E. \& Gava A. 2003. Intoxicação natural por Ateleia glazioviana (Leg.Papilionoideae) em ovinos. XI Encontro Nacional de Patologia Veterinária, Botucatu, SP. 60p.

Gava A., Cristani J., Branco J.V., Neves D.S., Mondadori A.J. \& Sousa R.S. 1998. Mortes súbitas em bovinos causadas pela ingestão de Mascagnia sp. (Malpighiaceae), no Estado de Santa Catarina. Pesq. Vet. Bras. 18(1):16-20. <http://dx.doi.org/10.1590/S0100-736X1998000100003>

Hayakawa I., Shioda S., Chinen T., Hatanaka T., Ebisu H., Sakakura A., Usui T. \& Kigoshi H. 2016. Discovery of 06 -benzyl glaziovianin A, a potent cytotoxic substance and a potent inhibitor of a,b-tubulin polymerization. Bioorganic Med. Chem. 24(21):5639-5645. <http://dx.doi.org/10.1016/j. bmc.2016.09.026><PMid:27665177>

Helayel M.A., França T.N., Seixas J.N., Nogueira V.A., Caldas S.A. \& Peixoto P.V. 2009. Morte súbita em bovinos causada pela ingestão de Pseudocalymma elegans (Bignoniaceae) no município de Rio Bonito, RJ. Pesq. Vet. Bras. 29(7):498-508. <http://dx.doi.org/10.1590/S0100-736X2009000700003> 
Helayel M.A., Caldas S.A., Peixoto T.C., França T.N., Tokarnia C.H., Döbereiner J., Nogueira V.A. \& Peixoto P.V. 2011. Antagonism of acetamid in experiments with sheep, goats and rabbits indicates that monofluoroacetate is the toxic principle of Pseudocalymma elegans Bignoniaceae. Pesq. Vet. Bras. 31(10):867-874. <http://dx.doi.org/10.1590/S0100-736X2011001000006>

Jabour F.F., Seixas J.N., Tokarnia C.H. \& Brito M.F. 2006. Variation of the toxicity of Arrabidaea bilabiata (Bignoniaceae) in rabbits. Pesq. Vet. Bras. 26(3):171-176. <http://dx.doi.org/10.1590/S0100-736X2006000300008>

Koch I., Rapini A., Simões A.O., Kinoshita L.S., Spina A.P. \& Castello A.C.D. 2015. Apocynaceae in Lista de Espécies da Flora do Brasil. Jardim Botânico do Rio de Janeiro. Disponivel em <http://floradobrasil.jbrj.gov.br/jabot/ floradobrasil/FB33737> Acesso em 12 jul. 2017.

Krebs H.C., Kemmerling W. \& Habermehl G. 1994. Qualitative and quantitative determination of fluoroacetic acid in Arrabidea bilabiata and Palicourea marcgravii by 19F-NMR spectroscopy. Toxicon 32(8):909-913. <http:// dx.doi.org/10.1016/0041-0101(94)90369-7><PMid:7985195>

Lago E.P., Melo M.M., Araújo R.B., Nascimento E.F., Silva E.F. \& Melo M.B. 2009. Perfis eletrocardiográfico e ecodopplercardiográfico de ovinos após ingestão da suspensão aquosa de Mascagnia rigida Griseb. (Malpighiaceae). Arq. Bras. Med. Vet. Zootec. 61(4):853-862. <http://dx.doi.org/10.1590/ S0102-09352009000400012>

Leániz J.A.B., Utaraviçius E.L. \& Aguirrezabala S.A. 2013. Estudio de la toxicidad de Nerium oleander en ovinos. Tesis de Grado, Universidad de La República. 61p.

Lee S.T., Cook D., Riet-Correa F., Pfister J.A., Anderson W.R., Lima F.G. \& Gardner D. 2012. Detection of monofluoracetate in Palicourea and Amorimia species. Toxicon 60(5):791-796. <http://dx.doi.org/10.1016/j.toxicon.2012.05.029> $<$ PMid:22699106>

Lemos R.A.A., Guimarães E.B., Carvalho N.M., Nogueira A.P.A., Santos B.S., Souza R.I.C., Cardinal S.G. \& Kassab H.O. 2011. Plant poisonings in Mato Grosso do Sul, p.68-72. In: Riet-Correa F., Pfister J., Schild A.L. \& Wierenga T. (Eds), Poisoning by Plants, Mycotoxins, and Related Toxins. CAB International, Wallingford, UK. <http://dx.doi.org/10.1079/9781845938338.0068>.

Lima E.F., Medeiros R.M., Cook D., Lee S.T., Kaehler M., Santos-Barbosa J.M. \& Riet-Correa F. 2016. Studies in regard to the classification and putative toxicity of Fridericia japurensis (Arrabidaea japurensis) in Brazil. Toxicon 115(1):22-27. <http://dx.doi.org/10.1016/j.toxicon.2016.03.001> $<$ PMid:26945838>

Lohmann L.G. 2015. Bignoniaceae in Lista de Espécies da Flora do Brasil. Jardim Botânico do Rio de Janeiro. Disponivel em <http://floradobrasil. jbrj.gov.br/jabot/floradobrasil/FB113368> Acesso em 12 jul. 2017.

Lohmann L.G. \& Taylor C.M. 2014. A new generic classification of tribe Bignonieae (Bignoniaceae) 1. Ann. Missouri Bot. Gard. 99(3):348-489. <http://dx.doi.org/10.3417/2003187>

Mamede M.C.H. 2015. Amorimia in Lista de Espécies da Flora do Brasil. Jardim Botânico do Rio de Janeiro. Disponível em <http://floradobrasil.jbrj.gov. br/jabot/floradobrasil/FB101437> Acesso em 7 jan. 2017.

Mansano V.F., Pinto R.B. \& Pennington T. 2015. Ateleia in Lista de Espécies da Flora do Brasil. Jardim Botânico do Rio de Janeiro. Disponível em <http://floradobrasil.jbrj.gov.br/jabot/floradobrasil/FB82648> Acesso em 7 jan. 2017.

McIlroy J.C. 1981. The sensivity of Australian animals to 1080 poison. I. Intraespecific variation and factors affecting acute toxicity. II. Marsupial and eutherian carnivores. Aust. Wildl. Res. 8(2):369-383. <http://dx.doi. org/10.1071/WR9810369>

Medeiros R.M.T., Geraldo Neto S.A., Barbosa R.C., Lima E.F. \& Riet-Correa F. 2002. Sudden bovine death from Mascagnia rigida in northeastern Brazil. Vet. Hum. Toxicol. 44(5):286-288. <PMid:12361113>

Nogueira V.A., França T.N. \& Peixoto P.V. 2009. Intoxicação por antibióticos ionóforos em animais. Pesq. Vet. Bras. 29(3):191-197. <http://dx.doi. org/10.1590/S0100-736X2009000300001>
Oliveira A.C., Oliveira G.C., Paraguassu A.A. \& Freire L.M.G.M. 1978. Intoxicação por um "tingui" (Mascagnia rigida Griseb.) em caprinos na Bahia. Anais XVI Congr. Bras. Med. Vet., Salvador, 1:172.

Oliveira-Neto T.S., Riet-Correa F., Lee S.T., Cook D., Barbosa F.M.S., SilvaNeto J.F., Simões S.V.D. \& Lucena R.B. 2017. Poisoning in goats by the monofluoracetate-containing plant Palicourea aeneofusca (Rubiaceae). Toxicon 135(1):12-16. <http://dx.doi.org/10.1016/j.toxicon.2017.05.025> <PMid:28576552>

Pacífico da Silva I. \& Soto-Blanco B. 2010. Conditioning taste aversion to Mascagniarigida (Malpighiaceae) in sheep. Res. Vet. Sci. 88(2):239-241. <http://dx.doi.org/10.1016/j.rvsc.2009.08.012> <PMid:19836034>

Pacífico da Silva I., Lira R.A., Barbosa R.R., Batista J.S. \& Soto-Blanco B. 2008. Intoxicação natural pelas folhas de Mascagnia rigida (Malpighiaceae) em ovinos. Arqs Inst. Biológico, São Paulo, 75(2):229-233.

Paraguassu A.A. 1983. Intoxicação experimental por Mascagnia rigida Grisebach (Malpighiaceae) em caprinos no Nordeste do Brasil. Dissertação de Mestrado em Medicina Veterinária, Curso de Pós-Graduação em Ciências Veterinárias, Universidade Federal Rural do Rio de Janeiro, RJ. 65p.

Pavarini S.P., Soares M.P., Bandarra P.M., Gomes D.C., Bandinelli M.B., Cruz C.E. \& Driemeier D. 2011. Mortes súbitas em bovinos causadas por Amorimia exotropica (Malpighiaceae) no Rio Grande do Sul. Pesq. Vet. Bras. 31(4):291296. <http://dx.doi.org/10.1590/S0100-736X2011000400004>

Pedroso P.M.O., Bandarra P.M., Bezerra Júnior P.S., Raymundo D.L., Borba M.R., Leal J.S. \& Driemeier D. 2009. Intoxicação natural e experimental por Nerium oleander (Apocynaceae) em bovinos no Rio Grande do Sul. Pesq. Vet. Bras. 29(5):404-408. <http://dx.doi.org/10.1590/S0100736X2009000500008>

Pedroza H.P. 2015. Psychotria hoffmannseggiana: uma nova espécie de planta tóxica para bovinos. Dissertação de Mestrado, Escola de Veterinária, Universidade Federal de Minas Gerais, Belo Horizonte, MG. 41p.

Pedroza H.P., Ferreira M.G., Carvalho J.G., Melo K.D.A., Keller K.M., Melo M.M. \& Soto-Blanco B. 2015. Concentrações de oleandrina nas folhas de Nerium oleander de diferentes cores da floração. Ciência Rural 45(5):864-866. <http://dx.doi.org/10.1590/0103-8478cr20140885>

Peixoto T.C., Oliveira L.I., Caldas S.A., Catunda Junior F.E.A., Carvalho M.G., França T.N. \& Peixoto P.V. 2011. Efeito protetor da acetamida sobre as intoxicações experimentais em ratos por monofluoroacetato de sódio e por algumas plantas brasileiras que causam morte súbita. Pesq. Vet. Bras. 31(11):938-952.<http://dx.doi.org/10.1590/S0100-736X2011001100002>

Raffi M.B., Rech R.R., Sallis E.S.V., Rodrigues A. \& Barros C.S.L. 2006. Chronic cardiomyopathy and encephalic spongy changes in sheep experimentally fed Ateleia glazioviana. Ciência Rural 36(6):1860-1866. <http://dx.doi. org/10.1590/S0103-84782006000600030>

Ribeiro D.P., Silva Filho G.B., Chaves H.A.S., Lemos B.O., Aguiar Filho C.R., Brito L.B., Almeida V.A. \& Mendonça F.S. 2016. Intoxicação espontânea por Kalanchoe blossfeldiana em bovinos. Pesq. Vet. Bras. 36(Supl. 2):90-91.

Riet-Correa F. \& Medeiros R.M. 2001. Intoxicações por plantas em ruminantes no Brasil e no Uruguai: importância econômica, controle e riscos para a saúde pública. Pesq. Vet. Bras. 21(1):38-42.<http://dx.doi.org/10.1590/ S0100-736X2001000100008>

Riet-Correa F. \& Méndez M.C. 2007. Plantas tóxicas e micotoxicoses, p.191193. In: Riet-Correa F., Schild A.L., Lemos R.A. \& Borges J.R.J. (Eds), Doenças de Ruminantes e Equinos. Vol.2. $3^{\mathrm{a}}$ ed. Pallotti, Santa Maria.

Riet-Correa F., Schild A.L., Mendez M.C. \& Lemos R.A.A. 2001. Doenças de Ruminantes e Equinos. Vol.2. Editora Varela, São Paulo, SP, p.279-283.

Riet-Correa G., Terra F.F., Schild A.L., Riet-Correa F. \& Barros S.S. 2005. Intoxicação experimental por Tetrapterys multiglandulosa (Malpighiaceae) em ovinos. Pesq.Vet. Bras. 25(2):91-96. <http://dx.doi.org/10.1590/ S0100-736X2005000200005>

Rissi D.R., Rech R.R., Pierezan F., Gabriel A.L., Trost M.E., Brum J.S., Kommers G.D. \& Barros C.S.L. 2007. Intoxicações por plantas e micotoxinas associadas 
a plantas em bovinos no Rio Grande do Sul: 461 casos. Pesq. Vet. Bras. 27(7):261-268. <http://dx.doi.org/10.1590/S0100-736X2007000700002>

Santos-Barbosa J.M., Lee S.T., Cook D., Gardner D.R., Viana L.H. \& Ré N. 2017. A gas chromatography-mass spectrometry method for the detection and quantitation of monofluoroacetate in plants toxic to livestock. J. Agricult. Food Chem. 65(7):1428-1433. <http://dx.doi.org/10.1021/acs. jafc.7b00294><PMid:28132508>

Schons S. 2011. Plantas tóxicas para ruminantes e equídeos na região central de Rondônia. Tese de Doutorado em Patologia, Universidade Federal de Pelotas, Pelotas. 78p.

Schons S.V., Mello T.L., Riet-Correa F. \& Schild AL. 2011. Poisoning by Amorimia (Mascagnia) septium in sheep in northern Brazil. Toxicon 57:781-786.

Silva D.M., Riet-Correa F., Medeiros R.M.T. \& Oliveira O.F. 2006. Plantas tóxicas para ruminantes e equídeos no Seridó Ocidental e Oriental do Rio Grande do Norte. Pesq. Vet. Bras. 26(4):223-236. <http://dx.doi.org/10.1590/ S0100-736X2006000400007>

Smith G. 2004. Toxicology brief: Kalanchoe species poisoning in pets. Vet. Med. 11(1):933-936.

Soares M.P., Pavarini S.P., Adrien M.L., Quevedo P.S., Schild A.L., Peixoto P.V., Cruz C.E.F. \& Driemeier D. 2011. Amorimia exotropica poisoning as a presumptive cause of myocardial fibrosis in cattle. J. Vet. Diagn. Invest. 23(6):1223-1229. <http://dx.doi.org/10.1177/1040638711425586> <PMid:22362807>

Soto-Blanco B., Fontenele-Neto J.D., Silva D.M., Reis P.F.C.C. \& Nóbrega J.E. 2006. Acute cattle intoxication from Nerium oleander pods. Trop. Anim. Health Prod. 38(6):351-354. <http://dx.doi.org/10.1007/s11250-0064400- $\mathrm{x}><$ PMid:17243471>

Stigger A.L., Barros C.S.L., Langohr I.M. \& Barros S.S. 2001. Intoxicação experimental por Ateleia glazioviana (Leg.Papilionoideae) em ovinos. Pesq. Vet. Bras. 21(3):98-108. <http://dx.doi.org/10.1590/S0100736X2001000300002>

Taylor C.M. 1996. Overview of the Psychotrieae (Rubiaceae) in the Neotropics. Opera Bot. Belg. 7:261-270.

Taylor C.M. 2007. Psychotria, p.389-412. In: Jung-Mendaçolli S. (Ed.), Rubiaceae: flora fanerogâmica do estado de São Paulo. Instituto de Botânica, São Paulo.

Taylor C.M. 2015a. Rubiacearum americanarum magna hama pars XXXIII: the new group Palicourea sect. Didymocarpae with four new species and two new subspecies (Palicoureeae). Novon, J. Bot. Nomenclature 23(4):452478. <http://dx.doi.org/10.3417/2012003>

Taylor C.M. 2015b. Rubiacearum americanarum magna hama pars XXXIV: The new group Palicourea sect. Tricephalium with eight new species and a new subspecies. Novon, J. Bot. Nomenclature 24(1):55-95. <http://dx.doi. org/10.3417/2015001>
Taylor C.M. \& Gereau R.E. 2013. The genus Carapichea (Rubiaceae: Psychotrieae). Ann. Missouri Bot. Gard. 99(1):100-127. <http://dx.doi. org/10.3417/2011064>

Taylor C.M., Lorence D.H. \& Gereau R.E. 2010. Rubiacearum americanarum magna hama pars XXV: the nocturnally flowering Psychotria domingensisCoussarea hondensis group plus three other Mesoamerican Psychotria species transfer to Palicourea. Novon, J. Bot. Nomenclature 20(4):481-492. $<$ http://dx.doi.org/10.3417/2009124>

Tokarnia C.H. \& Döbereiner J. 1981. Intoxicação por Arrabidaea japurensis (Bignoniaceae) em bovinos em Roraima. Pesq. Vet. Bras. 1(1):7-17.

Tokarnia C.H., Canella C.F.C. \& Döbereiner J. 1961. Intoxicação por um "tingui" (Mascagnia rigida Griseb.) em bovinos no Nordeste do Brasil. Arqs Inst. Biol. Anim. 4:203-215.

Tokarnia C.H., Döbereiner J. \& Peixoto P.V. 1985. Intoxicação por Mascagnia aff. rigida (Malpighiaceae) em bovinos no Norte do Espirito Santo. Pesq. Vet. Bras. 5(3):77-91.

Tokarnia C.H., Döbereiner J. \& Canella C.F.C. 1987. Intoxicação experimental por Mascagnia rigida (Malpighiaceae) em coelhos. Pesq. Vet. Bras. 7(1):11-16.

Tokarnia C.H., Peixoto P.V. \& Döbereiner J. 1993. Intoxicação experimental por Pseudocalymma elegans (Bignoniaceae) em caprinos. Pesq. Agropec. Bras. 13(1/2):35-39.

Tokarnia C.H., Döbereiner J. \& Peixoto P.V. 1994. Aspectos clínico-patológicos complementares da intoxicação por algumas plantas tóxicas brasileiras. Pesq. Vet. Bras. 14(4):111-122.

Tokarnia C.H., Döbereiner J., Couceiro J.E.M. \& Cordeiro Silva A.C. 1983. Intoxicação por Palicourea aeneofusca (Rubiaceae), A causa de mortes súbitas em bovinos na Zona da Mata Pernambucana. Pesq. Vet. Bras. 3(3):75-79.

Tokarnia C.H., Peixoto P.V., Döbereiner J., Consorte I.B. \& Gava A. 1989. Tetrapterys spp. (Malpighiaceae): a causa de mortandades em bovinos caracterizadas por alterações cardíacas. Pesq. Vet. Bras. 9:23-44

Tokarnia C.H., Brito M.F., Barbosa J.D., Peixoto P.V. \& Döbereiner J. 2012. Plantas que afetam o funcionamento do coração, p.27-94. In: Ibid. (Eds), Plantas Tóxicas do Brasil para Animais de Produção. $2^{\underline{a}}$ ed. Helianthus, Rio de Janeiro.

Vasconcelos J.S., Riet-Correa F., Dantas A.F., Medeiros R.M.T. \& Dantas A.J.A 2008a. Mortes súbitas em bovinos causadas por Palicourea aeneofusca (Rubiaceae) e Mascagnia rigida (Malpighiaceae) na Zona da Mata Paraibana. Pesq. Vet. Bras. 28(10):457-460. <http://dx.doi.org/10.1590/S0100736X2008001000003>

Vasconcelos J.S., Riet-Correa F., Dantas A.F.M., Medeiros R.M., Galiza G.J.N., Oliveira D.M. \& Pessoa A.F. 2008b. Intoxicação por Mascagnia rigida (Malpighiaceae) em ovinos e caprinos. Pesq. Vet. Bras. 28(10):521-526. <http://dx.doi.org/10.1590/S0100-736X2008001000013>

Zibbu G. \& Batra A. 2010. A review on chemistry and pharmacological activity of Nerium oleander L. J. Chem. Phar. Res. 2(6):351-358. 